\title{
Treatment effects of oxaliplatin combined with gemcitabine on colorectal cancer and its influence on HMGB1 expression
}

\author{
SHUANGSHUANG WEN ${ }^{1 *}$, XIAOPENG DU ${ }^{2 *}$, YANYAN GOU $^{3}$ and LIN JIANG ${ }^{4}$ \\ ${ }^{1}$ Department of Pharmacy, Yantaishan Hospital, Yantai, Shandong 264001; ${ }^{2}$ Healthcare Training Center, \\ Jinan Military General Hospital, Jinan, Shandong 250031; ${ }^{3}$ Dongying Center for Food and Drug Control, \\ Dongying, Shandong 257091; ${ }^{4}$ Department of Oncology, Hubei Provincial Corps Hospital of \\ The Chinese People's Armed Police Forces, Wuhan, Hubei 430060, P.R. China
}

Received May 5, 2016; Accepted August 18, 2016

DOI: $10.3892 / \mathrm{ol} .2016 .5053$

\begin{abstract}
In the present study, we analyzed the supra-additive effect of oxaliplatin in combination with gemcitabine on terminal colorectal cancer and its influence on high-mobility group box 1 (HMGB1) expression. A total of 86 patients with terminal colorectal cancer were enrolled in this study. Patients received oxaliplatin in combination with gemcitabine. Immunohistochemistry was used to determined the subcellular localization of HMGB1 in cancer tissues as well as in para-carcinoma tissues, and RT-PCR and western blot analysis were used to assess the mRNA and protein expression level, respectively. The total effective rate was analyzed based on WHO tumor chemotherapy reference where complete remission (CR) implies the complete disappearance of the tumor; partial remission (PR) implies that the tumor size was reduced at least by $50 \%$; stable disease (SD) implies that the tumor size remained unchanged and progressive disease (PD) implies an increase in the size of the tumor by $\geq 25 \%$. We identified 20 cases of CR, 37 cases of PR, 12 cases of SD and 17 cases of PD. The total effective rate (CR+PR) was $66.3 \%$. HMGB1 expression rate in the cancer tissues in the effective group was significantly lower than that in the ineffective group. Positive expression of HMGB1 protein was mainly localized in the karyon. Survival time in the patients with positive HMGB1 expression was significantly shorter than that in the patients with negative HMGB1 expression. In the effective group, HMGB1 mRNA and protein expression levels were obviously lower than those in the ineffective group. We conclude that the reduced expression rate of HMGB1 in terminal colorectal
\end{abstract}

Correspondence to: Dr Lin Jiang, Department of Oncology, Hubei Provincial Corps Hospital of Chinese People's Armed Police Forces, 475 Minzhu Road, Wuhan, Hubei 430060, P.R. China

E-mail: jiang_lin1212@163.com

${ }^{*}$ Contributed equally

Key words: oxaliplatin, gemcitabine, terminal colorectal cancer, high-mobility group box 1, HMGB1 cancer cases was probably related to the effects of oxaliplatin combined with gemcitabine.

\section{Introduction}

Every year there are 0.9 million new colorectal cancer cases worldwide (1), which accounts for $8.7 \%$ of all new cancer cases. In USA, colorectal cancer has the second highest mortality rate after lung cancer, and in China it ranks third.

Resectability of colorectal cancer is only indicated in $35-48 \%$ of cases and the possibility of recurrence and metastasis is $\sim 55 \%$ (2). Chemotherapy is typically the main treatment for terminal colorectal cancer. Oxaliplatin is a third generation platinum-containing anticancer drug after cis-platinum and carboplatin with less side effects and better treatment effects (3). Gemcitabine (a vidarabine analogue) is a cell cycle-specific drug with satisfactory effects on malignant tumors of the digestive system (4). Overexpression of high-mobility group box 1 (HMGB1) has been shown in various cancers such as lung, colorectal and gastric tumors (5). HMGB1 might be implicated in tumor formation, tumor development, tumor infiltration and tumor transfer and might influence the outcome of chemotherapy (5). HMGB1 inhibitors, such as the anti-HMGB1 antibody, soluble terminal receptor for advanced glycation end-products (RAGE) and small interfering RNA (siRNA) can improve the curative effect of chemotherapy through inhibition of HMGB1 expression (6). This study was conducted to verify any possible impact that oxaliplatin combined with gemcitabine might have on the expression of HMGB1 in terminal colorectal cancer patients.

\section{Patients and methods}

Patients. From January 2014 to June 2015, 86 terminal colorectal cancer patients were enrolled in this study. None of these cases had received any type of cancer treatment prior to their enrollment. The estimated survival time of the patients was $\sim 12$ months. After enrollment, the patients were treated with oxaliplatin combined with gemcitabine and all patients completed the course of treatment even those who suffered from severe side effects. Karnofsky performance 
status scoring was $>70$ points; hemoglobin level was $\geq 90 \mathrm{~g} / 1$, neutrophil differential count was $\geq 2.0 \times 10^{9} / 1$, blood platelet count was $\geq 100 \times 10^{9} / 1$; glutamic-pyruvic transaminase was within normal range and no severe organ dysfunction was reported. The patients included 50 males and 36 females aged from 48 to 73 years (average age, $57.6 \pm 13.3$ years). According to Dukes' staging system, 57 patients were in stage $\mathrm{C} 2$ and 29 cases were in stage $\mathrm{D}$, while adenocarcinoma was the most common histologic type. The maximum diameter of tumors ranged from 3.0 to $6.2 \mathrm{~cm}$ (average, $5.3 \pm 1.4 \mathrm{~cm}$ ). There were 66 cases with lymphatic metastasis, 13 cases with liver metastasis and 7 cases with spread of cancer to the lung and other parts of the body. This study was approved by the ethics committees of the participating hospitals, and all patients and their family members provided conformed consent.

Treatment method. We used the following doses of gemcitabine and oxaliplatin: the gemcitabine dose was $1,000 \mathrm{mg} / \mathrm{m}^{2} \mathrm{ivgtt}$ in $100 \mathrm{ml}$ normal saline for $30-60 \mathrm{~min}$ at days 1 and 8 of the cycle; and oxaliplatin: $100 \mathrm{mg} / \mathrm{m}^{2} \mathrm{ivgtt}$ for $2 \mathrm{~h}$ at day 2 of the cycle. There were 21 days in each cycle and patients were treated for 2 cycles under close observation. For severe stenosis of bowel and adhesion, we performed minimally invasive palliative surgery for resection and colorectal colonoscopy was conducted for tissue sampling. All procedures were conducted following patient consent. The follow-up visits continued for 18 months.

Observation index and testing method. The total effective rate was analyzed based on WHO tumor chemotherapy reference where complete remission (CR) implies complete disappearance of the tumor, partial remission (PR) implies that the tumor size was reduced at least by $50 \%$, stable disease (SD) implies that the tumor size remained unchanged and progressive disease (PD) implies an increase in the size of the tumor by $\geq 25 \%$. CT scan examinations were conducted to measure the size of the tumors. Immunohistochemistry was used to study the subcellular localization of HMGB1 in the cancer tissues as well as the para-carcinoma tissues $(\geq 10 \mathrm{~cm}$ away from the tumor edge). RT-PCR and western blotting were used to assess the mRNAand protein expression level, respectively. Conventional method was used for tissue preparation as follows. The samples were dewaxed using xylene, washed with gradient alcohol, stained with hematoxylin, dehydrated by ethyl alcohol, and the samples were sealed by neutral resins followed by washing with phosphate-buffered saline (PBS). We added induced antigen retrieval solution and goat serum blocking reagent. The primary antibody was then added followed by washing and addition of the secondary antibody (R\&D Systems, Inc., Minneapolis, MN, USA). Samples were washed with PBS and streptavidin peroxidase was added and then DAB color developing agent was added after PBS washing and counterstaining was carried out using hematoxylin. Intensity of staining (IHS) was calculated using the following formula: IHS = A x B, where 'A' is the number of positive cells and ' $\mathrm{B}$ ' is the intensity of the color. Positive expression was considered at IHS of 3 .

RT-PCR. Conventional TRIzol reagent method was used for RNA extraction and ultraviolet absorption spectroscopy method was used to verify the concentration and purity.
SuperScript ${ }^{\mathrm{TM}}$ III Reverse Transcriptase kit (ABI, Invitrogen Life Technologies, Carlsbad, CA, USA) was used for cDNA synthesis and Primer 3.0 online software was used to design the primers: HMGB1 (F), 5'-ATATGGCAAAAGCGGAC AAG-3' and HMGB1 (R), 5'-AGGCCAGGATGTTCTCC TTT-3'. The internal control was $\beta$-actin (F), 5'-CTCTGG CCGTACCACTGGC-3', and $\beta$-actin (R), 5'-GTGAAGCTG TAGCCGCGC. The reaction system used was the following: $1 \mu \mathrm{l}$ of cDNA, $2 \mu \mathrm{l}$ of $10 \mathrm{X}$ buffer, $1 \mu \mathrm{l}$ of $\mathrm{Mg}^{2+}, 1 \mu \mathrm{l}$ assay and $0.2 \mu \mathrm{l} \mathrm{Taq}$ and $14.8 \mu \mathrm{l}$ of $\mathrm{ddH}_{2} \mathrm{O}$. We used ABI $7500 \mathrm{qPCR}$ apparatus and the parameters were: $95^{\circ} \mathrm{C}$ for $2 \mathrm{~min}, 94^{\circ} \mathrm{C}$ for $20 \mathrm{sec}, 60^{\circ} \mathrm{C}$ for $20 \mathrm{sec}$ and $72^{\circ} \mathrm{C}$ for $30 \mathrm{sec}$ for a total of 40 cycles. Fluorescent quantitation PCR was used to monitor the expression of HMGB1 mRNA, and $\mathrm{Ct}$ in all samples was tested using fold $=2^{-\Delta \Delta \mathrm{Ct}}$ to express the multiple proportion relation between the target gene and internal control gene. Samples were testing 3 times and the average values were recorded.

Western blot analysis. The HMGB1 antibody was purchased from Abcam Company, the secondary antibody was obtained from R\&D Systems, Inc., and the $\beta$-actin antibody was purchased from Santa Cruz Biotechnology, Inc. (Santa Cruz, CA, USA). Trans-blot was obtained from Bio-Rad (Berkeley, CA, USA). For imaging, we used a gel image analysis system (UVP Co., San Gabriel, CA, USA). Conventional RIPA lysate was used to extract total protein, and the BCA protein quantification kit was purchased from Pierce Biotechnology, Inc. (Rockford, IL, USA). The concentration was assessed using a microplate reader. Proteins were separated using SDS-PAGE, and the separated proteins were transferred and blotted onto a polyvinylidene fluoride membrane. Next, the membranes were blocked and chemiluminescence was performed.

Statistical analysis. We used the Statistical Package for Social Sciences (SPSS, Inc., Chicago, IL, USA) for our statistical analyses. Data are presented as mean \pm standard deviation (SD) and proportions. Continuous data are presented as mean \pm SD. Categorical values were evaluated using the Chi-square test. Survival was analyzed by Kaplan-Meier method. $\mathrm{P}<0.05$ was considered to indicate a statistically significant difference.

\section{Results}

Analysis of the chemotherapeutic effects. We identified 20 cases of CR, 37 cases of PR, 12 cases of SD and 17 cases of PD. The total effective rate $(\mathrm{CR}+\mathrm{PR})$ was $66.3 \%$. Positive expression of HMGB1 protein was mainly localized in the karyon (light yellow and dark yellow particles). In the effective group, we observed 12 cases $(21.1 \%)$ of positive expression of HMGB1 in the cancer tissues while in the ineffective group the number of positive cases was 12 (41.4\%). HMGB1 expression in the cancer tissues obtained from the effective group was significantly lower than that in the ineffective group $\left(\chi^{2}=3.947\right.$, $\mathrm{P}=0.047$ ). In the effective group, positive expression of HMGB1 in the para-carcinoma tissues was detected in 5 cases $(8.8 \%)$ while there were 3 cases in the ineffective group (10.3\%) and the difference was statistically significant $\left(\chi^{2}<0.001, \mathrm{P}=1.000\right)$ (Fig. 1). Survival time in the HMGB1-positive cases was obviously shorter than that in the negative cases (log-rank test $\chi^{2}=65.384, P<0.001$; Fig. 2). 

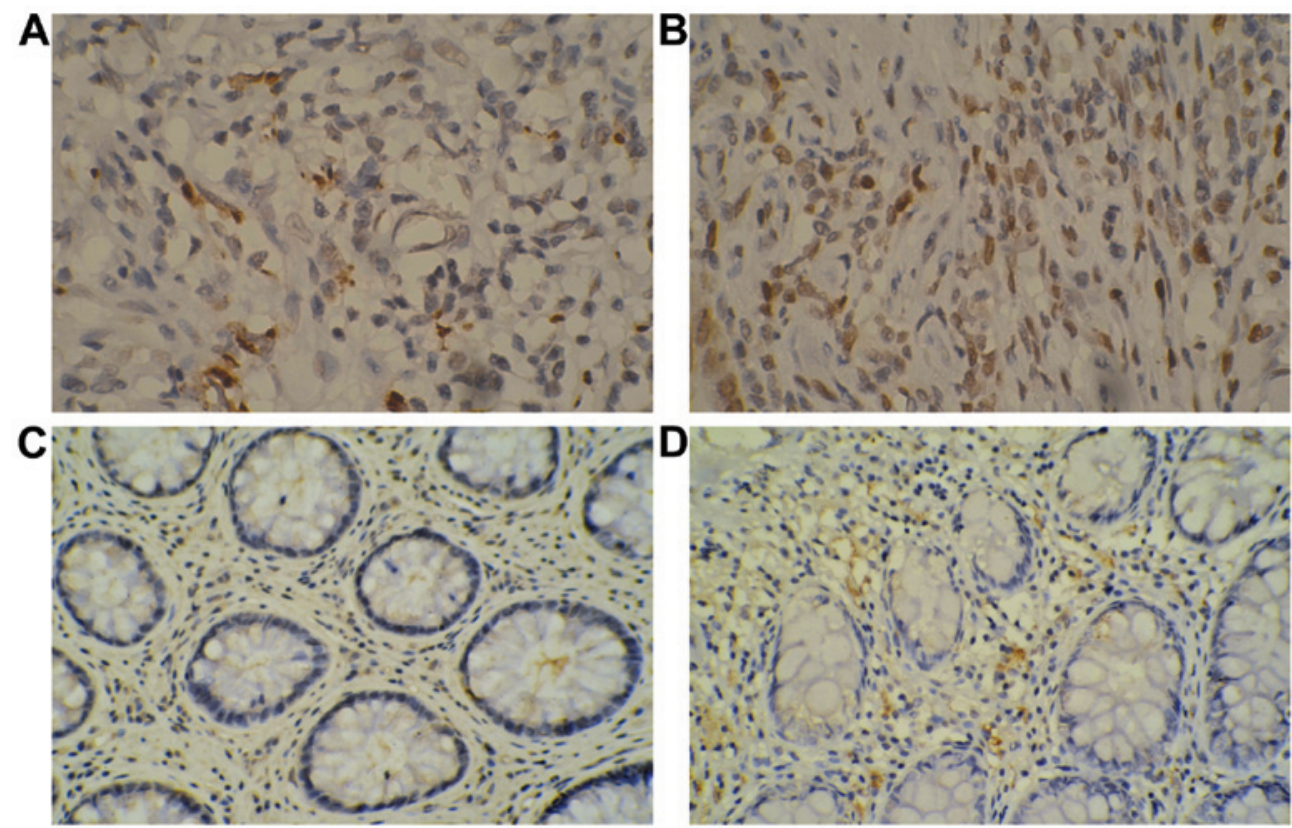

Figure 1. Immumohistochemical staining of high-mobility group box 1 (HMGB1) (x400 magnification). (A) Cancer tissue from the effective group, (B) cancer tissue from the ineffective group, (C) para-carcinoma tissue from the effective group, and (D) para-carcinoma tissue from the ineffective group.

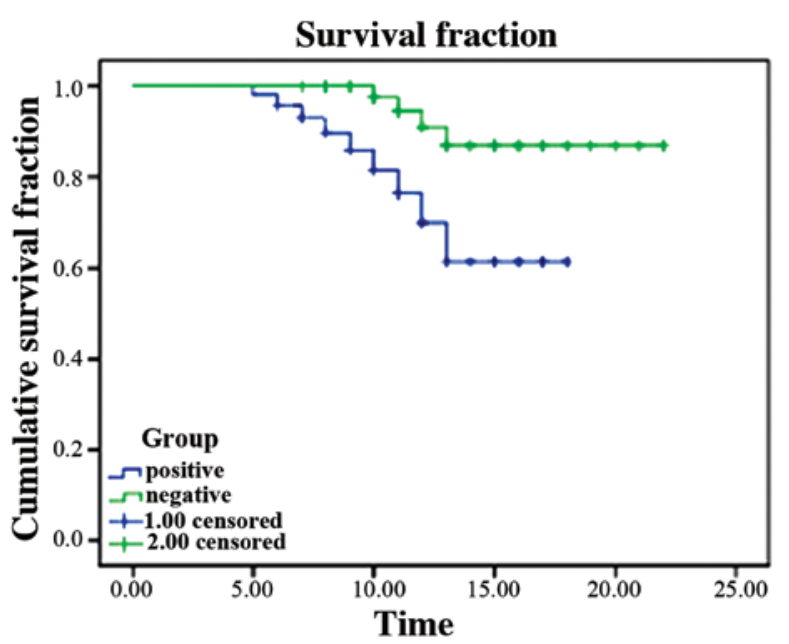

Figure 2. Survival analysis of colorectal cancer patients with high-mobility group box 1 (HMGB1)-positive expression compared with patients with HMGB1-negaive expresssion.

Comparison of $m R N A$ and protein expression levels. In the effective group, the HMGB1 mRNA expression level was obviously lower than that in the ineffective group $(0.16 \pm 0.02$ vs. $0.44 \pm 0.03, \mathrm{t}=8.246, \mathrm{P}<0.001)$, and also, the protein expression level was lower in the effective group than that in the ineffective group; differences were statistically significant ( $\mathrm{P}<0.05$; Fig. 3).

\section{Discussion}

Non-histones are highly conserved proteins and major components of chromosomes. Non-histone proteins can be divided into three families: HMGA, HMGB and HMGN. The HMGB1 gene is located on chromosome 13q12 and HMGB1 protein is

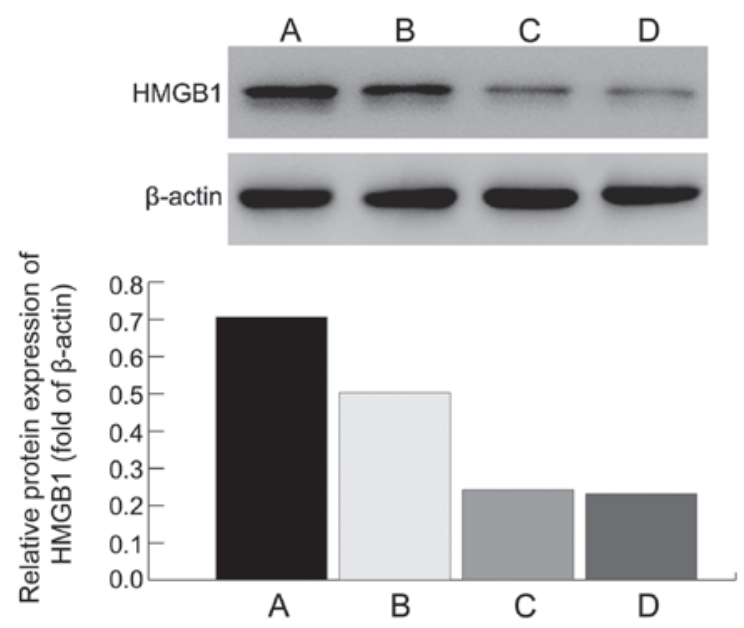

Figure 3. High-mobility group box 1 (HMGB1) protein expression as assessed by western blotting. Lane A, cancer tissue in ineffective group; lane B, cancer tissue in effective group; lane C, para-carcinoma tissue in ineffective group; lane D, para-carcinoma tissue in effective group.

mainly located inside the cell nucleus attached to DNA and is involved in gene transfer, recombination, repair, construction and stabilization of nucleosomes (7). Extracellular HMGB1 can combine with RAGE and the Toll-like receptor. Elevated levels of RAGE have been shown to be associated with poor prognosis in many types of tumors (8). Following engagement with extracellular HMGB1, RAGE internalizes and promotes the survival and migration of cancer cells through activation of nuclear factor $(\mathrm{NF})-\kappa \mathrm{B}$. This can also induce intracellular signal transduction and mediate an inflammatory response, cell proliferation, differentiation and metastasis (8). In colorectal cancer patients, HMGB1 overexpression has been shown to be positively correlated to tumor infiltration, lymphatic metastasis, distant metastasis, survival time and Dukes' staging (9). 
After blocking HMGB1 synthesis using siRNA, a significant reduction in invasion ability was reported in human colorectal cancer cell line SW620 (10). HMGB1 must be phosphorylated for secretion and HMGB1 phosphorylation is accomplished by protein kinase $\mathrm{C}$ (cPKC) and is secreted by a calcium-dependent mechanism (10).

Results obtained from a study conducted on serum HMGB1 levels in 219 colorectal cancer patients and 75 healthy cases, revealed that the serum HMGB1 level in colorectal cancer patients was increased by 1.5 -fold, which was similar to the diagnostic efficiency of carcinoembryonic antigen (CEA) in colorectal cancer. Diagnostic accuracy of HMGB1 index in stage I colorectal cancer was significantly better than that of CEA, however, the serum HMGB1 level was not related to the prognosis of colorectal cancer patients.

The functional mechanism of gemcitabine involves its transformation into difluorodeoxygenation cytidine triphosphate within the body through metabolism, consequently combining with cell DNA to break it and prevent cells from progressing from $\mathrm{G} 1$ to $\mathrm{S}$ phase of the cell cycle, thus, killing cells and inducing apoptosis (11). Compared with cytosine arabinoside, it is more difficult to be removed and it has better membrane penetrability and longer residence time in cells. It is more tolerable for patients as it has few unpleasant side effects (4). Compared with cis-platinum, oxaliplatin has broader antitumor activity and better treatment effects with no cross resistance (12). In the effective group, HMGB1 expression in cancer tissues was obviously lower than that in the ineffective group. HMGB1 expression in para-carcinoma tissues in the two groups demonstrated very small and insignificant differences. Survival time of the patients with HMGB1-positive expression was shorter than that found in patients with negative expression. In the effective group, HMGB1 mRNA and protein expression levels were lower than those in the ineffective group and differences were statistically significant. In conclusion, low expression of HMGB1 in terminal colorectal cancer may be related to treatment effects of oxaliplatin combined with gemcitabine.

\section{References}

1. Jemal A, Siegel R, Ward E, Murray T, Xu J and Thun MJ: Cancer statistics, 2007. CA Cancer J Clin 57: 43-66, 2007.

2. Bacchus CM, Dunfield L, Connor Gorber S, Holmes NM, Birtwhistle R, Dickinson JA, Lewin G, Singh H, Klarenbach S, Mai V, et al: Recommendations on screening for colorectal cancer in primary care. CMAJ 22: 13-15, 2016 .

3. van Hazel GA, Heinemann V, Sharma NK, Findlay MP, Ricke J, Peeters M, Perez D, Robinson BA, Strickland AH, Ferguson T, et al: SIRFLOX: Randomized phase III trial comparing first-line mFOLFOX6 (plus or minus bevacizumab) versus mFOLFOX6 (plus or minus bevacizumab) plus selective internal radiation therapy in patients with metastatic colorectal cancer. J Clin Oncol 34: 1723-1731, 2016.

4. Bai M, Deng T, Han R, Zhou L and Ba Y: Gemcitabine plus S-1 versus cetuximab as a third-line therapy in metastatic colorectal cancer: An observational trial. Int J Clin Exp Med 8: 21159-21165, 2015.

5. Sims GP, Rowe DC, Rietdijk ST, Herbst R and Coyle AJ: HMGB1 and RAGE in inflammation and cancer. Annu Rev Immunol 28: 367-388, 2010.

6. Stros M: HMGB proteins: Interactions with DNA and chromatin. Biochim Biophys Acta 1799: 101-113, 2010.

7. Tang D, Kang R, Zeh HJ III and Lotze MT: High-mobility group box 1 and cancer. Biochim Biophys Acta 1799: 131-140, 2010.

8. Kang JW, Koh EJ and Lee SM: Melatonin protects liver against ischemia and reperfusion injury through inhibition of toll-like receptor signaling pathway. J Pineal Res 50: 403-411, 2011.

9. Yao X, Zhao G, Yang H, Hong X, Bie L and Liu G: Overexpression of high-mobility group box 1 correlates with tumor progression and poor prognosis in human colorectal carcinoma. J Cancer Res Clin Oncol 136: 677-684, 2010.

10. Lee H, Park M, Shin N, Kim G, Kim YG, Shin JS and Kim H: High mobility group box-1 is phosphorylated by protein kinase $\mathrm{C}$ zeta and secreted in colon cancer cells. Biochem Biophys Res Commun 424: 321-326, 2012.

11. Hosokawa M, Saito M, Nakano A, Iwashita S, Ishizaka A Ueda K and Iwakawa S: Acquired resistance to decitabine and cross-resistance to gemcitabine during the long-term treatment of human HCT116 colorectal cancer cells with decitabine. Oncol Lett 10: 761-767, 2015.

12. Hosokawa Y, Watanabe M, Makino H, Mushiake H, Katsumata K, Maruno K, Fujino S and Sugiyama Y: Serum type IV collagen concentration correlates with indocyanine green retention rate and is an indicator of hepatotoxicity in patients receiving FOLFOX for colorectal cancer. Hepatogastroenterology 62: 653-656, 2015. 